Marquette University

e-Publications@Marquette

\title{
The Role of Loneliness as a Mediator Between Autism Features and Mental Health Among Autistic Young Adults
}

Hillary Schiltz

Marquette University

Alana J. McVey

Marquette University

Bridget Dolan Wozniak

Ascension Medical Group

Angela Haendel

Concordia University - Wisconsin

Rachel Stanley

Marquette University

See next page for additional authors

Follow this and additional works at: https://epublications.marquette.edu/psych_fac

Part of the Psychology Commons

\section{Recommended Citation}

Schiltz, Hillary; McVey, Alana J.; Wozniak, Bridget Dolan; Haendel, Angela; Stanley, Rachel; Arias, Alexis; Gordon, Nakia; and Van Hecke, Amy V., "The Role of Loneliness as a Mediator Between Autism Features and Mental Health Among Autistic Young Adults" (2021). Psychology Faculty Research and Publications. 517.

https://epublications.marquette.edu/psych_fac/517 


\section{Authors}

Hillary Schiltz, Alana J. McVey, Bridget Dolan Wozniak, Angela Haendel, Rachel Stanley, Alexis Arias, Nakia Gordon, and Amy V. Van Hecke 
Marquette University

e-Publications@Marquette

Psychology Faculty Research and Publications/College of Arts and Sciences

This paper is NOT THE PUBLISHED VERSION.

Access the published version via the link in the citation below.

Autism, Vol. 25, No. 2 (February 1, 2021): 545-555. DOI. This article is (C) SAGE Publications and permission has been granted for this version to appear in e-Publications@Marquette. SAGE Publications does not grant permission for this article to be further copied/distributed or hosted elsewhere without the express permission from SAGE Publications.

\section{The role of loneliness as a mediator between autism features and mental health among autistic young adults}

Hillary K. Schiltz

Marquette University

Alana J. McVey

Marquette University

University of California, Los Angeles

Bridget Dolan Wozniak

Ascension Medical Group

Angela D. Haendel

Concordia University Wisconsin

Rachel Stanley

Marquette University

Alexis Arias

Marquette University

Nakia Gordon 
Marquette University

\title{
Amy Vaughan Van Hecke
}

Marquette University

\begin{abstract}
Autistic adults commonly experience anxiety and depression. These mental health concerns are often tied to social experiences, such that mental well-being can be supported by social connection and deteriorated by loneliness. The mediating role of social and emotional loneliness (i.e. social isolation and lack of emotional attachment, respectively) between autism features and mental health has yet to be empirically tested among autistic adults. Here, 69 autistic young adults completed self-report questionnaires assessing social contact (Friendship Questionnaire), autism features (Autism Quotient), mental health (Liebowitz Social Anxiety Scale, Social Phobia Inventory, Beck Depression Inventory), and loneliness (Social and Emotional Loneliness Scale for Adults). Positive associations emerged between autism features, social loneliness, family loneliness, social anxiety, and depression. In addition, more social contact was related to less social and family loneliness and less social anxiety but was not related to depression. Mediation analyses indicated significant indirect effects of social contact and autism features on mental health through social loneliness. Indirect effects partially held substituting family loneliness for social loneliness and did not hold using romantic loneliness. In light of these results, the scientific and clinical implications of the role of loneliness for autistic young adults are discussed and recommendations provided.
\end{abstract}

\section{Lay abstract}

Autistic adults commonly experience mental health concerns including social anxiety and depression, which can have negative effects on their quality of life. It is not completely clear, however, why rates of mental health concerns are so high. Some evidence suggests that social connectedness might play a key role. The goal of this study was to explore links between loneliness, mental health concerns, autism features, and social contact among autistic adults and test whether the links between mental health with autism features and social contact can be explained by loneliness. Researchers in this study collected data using questionnaires completed by 69 autistic young adults. Autistic adults who reported more autism features also reported more social and family loneliness, higher levels of social anxiety and depression, and fewer initiated social contacts. In addition, adults with more social contact initiations were likely to report lower levels of social and family loneliness and social anxiety but not depression. Results showed that the link from social engagement and autism features to social anxiety and depression symptoms could be mostly explained by loneliness. The results of this study expand previous findings by illustrating one factor (loneliness) that might be responsible for the high rates of mental health concerns among adults on the autism spectrum. These findings highlight the importance of studying factors related to mental health concerns among autistic adults and ways to best support social connectedness for the mental well-being of autistic young adults.

\section{Keywords}

autism, friendship, loneliness, mental health

Mental health concerns are common for people on the autism spectrum (Hollocks et al., 2019). In particular, anxiety and depression occur in about $33 \%$ to $50 \%$ of autistic adults (Hofvander et al., 2009; Maddox \& White, 2015; Uljarević et al., 2020), with a notable increase in symptoms from adolescence to middle adulthood (Uljarević et al., 2020). In contrast, approximately $33.7 \%$ and $20.6 \%$ of neurotypical adults experience clinical levels of anxiety (Bandelow \& Michaelis, 2015) or depression (Hasin et al., 2018), respectively, at some point in 
their lives. Social anxiety, characterized by excessive fear of negative evaluation in social situations (American Psychiatric Association [APA], 2013), is one of the most common presentations of anxiety in autistic adults (Hollocks et al., 2019). Despite these high rates in autism, little work has explored factors that may precipitate mental health concerns among autistic people, especially in adulthood. It is particularly important to consider the mental health concerns of autistic young adults, given transitions experienced during this developmental stage, a greater need for services (Turcotte et al., 2016), and the "service cliff" many autistic young adults experience (Shattuck et al., 2011). Better understanding the factors related to mental health concerns among autistic young adults may bolster advocacy for the delivery of needed services.

Researchers have identified positive links between autism features and mental health concerns. Scores on a measure of autism symptoms have been shown to be higher among autistic youth with symptoms of anxiety compared to those without (Factor et al., 2017; McVey et al., 2018). Given the core social component of autism (APA, 2013), social anxiety may be rooted in autism-specific risk factors including social confusion, withdrawal, and negative social interactions (Bellini, 2006; Spain et al., 2018; Wood \& Gadow, 2010), and thus, ties between social anxiety and autism features have also been found among autistic adults (Bejerot et al., 2014). A similar pattern has emerged for depressive symptoms, namely, parent-report of autism features has been positively associated with depression (Mayes et al., 2011), and perceived group membership has been negatively associated with depressive symptoms (Hedley \& Young, 2006). The link between greater social difficulties and increased mental health concerns is not unique to autistic youth and adults; neurotypical young adults with higher scores on questionnaires assessing autism features (the Social Responsiveness Scale and Autism Quotient) have demonstrated more anxious and depressive symptoms (Kanne et al., 2009; Reed et al., 2016). Neurotypical adolescents with social anxiety, who commonly struggle socially (Spence \& Rapee, 2016), also demonstrate a greater likelihood of developing depression (Stein et al., 2001). This link between social challenges and mental health concerns may be operating through secondary factors. Evidence from neurotypical young adults has demonstrated loneliness as a mediator between features of autism and mental health concerns (generalized anxiety and depression; Stice \& Lavner, 2019), which raises the question, is the same process at play for autistic adults?

Psychological health and quality of life are tied to social experiences among neurotypical young adults (Reed et al., 2016). Likewise, well-being for adults on the autism spectrum is promoted by social acceptance (Kapp, 2018), friendship (Mazurek \& Kanne, 2010), and social support (Renty \& Roeyers, 2006), and undermined by feelings of loneliness (Hedley et al., 2018; White \& Roberson-Nay, 2009), lack of perceived group membership and downward social comparison (Hedley \& Young, 2006), and victimization (Hong et al., 2016). These experiences do not tend to improve over time, and, in fact, bullying may worsen with age for autistic people (Morton et al., 2019). Autistic people commonly experience social rejection and ostracism (Kapp, 2018) (even from their siblings; Toseeb et al., 2020), challenges in the development and maintenance of friendships (O'Hagan \& Hebron, 2017; Sosnowy et al., 2019), and poorer friendship quality (Bauminger \& Kasari, 2000; Locke et al., 2010). These experiences may elicit loneliness (Bauminger \& Kasari, 2000; Locke et al., 2010) and, in turn, contribute to poorer mental health (Hedley et al., 2018; White \& Roberson-Nay, 2009).

Although understudied among autistic adults, recent evidence points to more loneliness in this population compared to non-autistic adults (Ee et al., 2019) with more autism features linked with greater levels of loneliness (Ee et al., 2019; Hedley et al., 2018; Mazurek, 2014; Syu \& Lin, 2018). Qualitative evidence has indicated that sensory (i.e. auditory) and historical (i.e. prior negative peer interactions) factors may impede social connection, contributing to loneliness (Ee et al., 2019). In fact, sensory avoidance has been empirically linked with loneliness among autistic adults (Syu \& Lin, 2018). Consistent with these studies of loneliness in autism, many have considered loneliness to be a unidimensional construct (Peplau, 1982), yet others have suggested that loneliness is multidimensional (Weiss, 1973). From a multidimensional perspective, social 
loneliness, due to social isolation and lack of social relationships, is distinct from emotional loneliness, which is due to a lack of attachment (DiTommaso \& Spinner, 1993; Weiss, 1973). In addition, evidence exists for a further delineation between two dimensions of emotional loneliness: romantic and family loneliness (DiTommaso \& Spinner, 1993). To the authors' knowledge, however, these specific dimensions of loneliness have only explored as intervention outcomes among autistic adults (McVey et al., 2016).

Despite the high level of loneliness among autistic adults, the toll of loneliness on mental health has received little investigation. One study of 71 autistic adults (ages 17-56; $M=26.14 ; S D=8.20$ ) identified that loneliness was related to self-harm through depressive symptoms (Hedley et al., 2018), although the mediation analyses did not account for autism features. Another study of autistic adults ( $n=108$; ages $18-62 ; M=32.40 ; S D=12.50)$ found that loneliness and number of friends each explained unique variance in generalized anxiety and depression symptoms after controlling for characteristics of autism (Mazurek, 2014). Finally, a recent study also identified univariate associations between loneliness with autism features and mental health (generalized anxiety and depressive symptoms; Ee et al., 2019). While these three studies provide preliminary evidence that loneliness is linked with the mental health of autistic adults, no existing study has differentiated between the various types of loneliness in autism (i.e. social and emotional loneliness). Therefore, replication and extension of these findings is critical for elucidating the specific role that types of loneliness play in contributing to social anxiety and depression for autistic adults.

\section{Summary and aims}

Given the high prevalence of mental health concerns in autistic adults, exploring factors that may account for these rates is of paramount importance. Literature points to strong ties between social experiences and mental health outcomes in both neurotypical and autistic adults, yet the role of loneliness as a mediator between social experiences and mental health has only been tested among neurotypical adults and has focused on generalized anxiety symptoms. Here, we explored the links between features of autism, mental health concerns (social anxiety and depression symptoms), and loneliness among young adults on the autism spectrum. We further tested the indirect effects of both autism features and social contact on mental health through loneliness. We hypothesized that there would be positive associations among autism features, mental health (social anxiety and depressive symptoms), and loneliness, which would each be negatively related to social contact. In addition, we hypothesized that loneliness would mediate the links between features of autism/social contacts, and social anxiety and depressive symptoms (Figure 1). Given the lack of research on the social and emotional components of loneliness in autism, no specific hypotheses were generated for these distinct constructs.

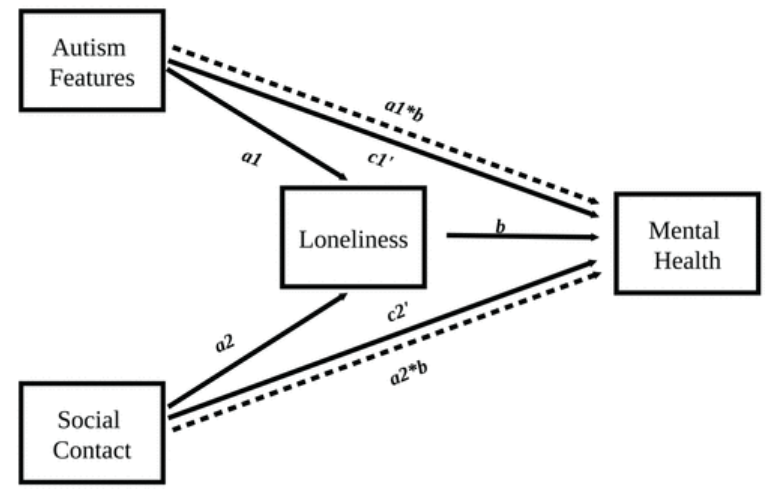

Figure 1. Proposed Mediation Model.

Note. Solid lines indicate direct effects. Dotted lines indicate indirect effects. 


\section{Method}

\section{Participants and procedure}

This study comprised data from 69 autistic young adults (ages 17-28) who completed a battery of questionnaires assessing social and emotional behavior as part of a larger randomized clinical trial of the Program for the Education and Enrichment of Relational Skills (PEERS ${ }^{\circ}$ ) for Young Adults (Laugeson, 2017) social skills intervention (registered with ClinicalTrials.gov, Identifier: NCT02680015). A portion of the data collected prior to the intervention was used in the present analyses. The Institutional Review Board at Marquette University approved the overarching study. Young adults were recruited from local autism clinics and support groups, referred by mental health professionals and word-of-mouth from prior participants, and from an inhouse waiting list at Marquette University. Inclusion criteria included adult diagnosed with autism between the ages of 17 and 28, parent-reported social challenges, English speaking, no history of major mental illness (i.e. bipolar disorder, schizophrenia, or psychosis), no physical conditions that would prohibit participation in the intervention (e.g. deafness, blindness, or physical disability). Additional details on recruitment and inclusion criteria for the larger study are detailed in the efficacy trial (McVey et al., 2016). For those who met screening criteria, informed consent was obtained, autism diagnosis was confirmed using the Autism Diagnostic Observation Schedule, Generic (ADOS-G; Lord et al., 2001), and IQ was estimated with the Kaufman Brief Intelligence Test, Second Edition (KBIT-2; Kaufman \& Kaufman, 2004). Trained research assistants administered these assessments-only those trained to research reliability in the lab administered the ADOS-G. Demographic characteristics of the sample are presented in Table 1. Young adults in this study were predominantly White males with verbal ability from well-educated, middle- to upper-socioeconomic status households. Community members were not involved in this study.

Table 1. Demographic characteristics.

\begin{tabular}{|l|c|c|}
\hline$n=69$ & & \\
\hline & $M(S D)$ & Range \\
\hline Age & $20.24(2.77)$ & $17-29$ \\
\hline KBIT-2 composite IQ & $96.56(17.79)$ & $63-145$ \\
\hline ADOS-G total & $11.72(3.46)$ & $7-21$ \\
\hline Gender & & \\
\hline \% Male & 81.00 & \\
\hline \% Female & 19.00 & \\
\hline Race & & \\
\hline \% White & 85.50 & \\
\hline \% Asian & 5.80 & \\
\hline \% Black & 4.40 & \\
\hline \% Middle Eastern & 1.40 & \\
\hline \% American Indian & 1.40 & \\
\hline \% Not reported & 1.40 & \\
\hline Ethnicity & & \\
\hline \% Non-Hispanic/Latinx & 88.40 & \\
\hline \% Hispanic/Latinx & 7.20 & \\
\hline \% Not reported & 4.40 & \\
\hline Household income & & \\
\hline \% Under 25K & 4.40 & \\
\hline$\%$ 25-50K & 14.50 & \\
\hline \% 50-75K & 21.70 & \\
\hline \% 75-100K & 18.80 & \\
\hline \% 100K+ & 39.10 & \\
\hline \% Not reported & 1.40 & \\
\hline & & \\
\hline
\end{tabular}


KBIT-2: Kaufman Brief Intelligence Test, Second Edition; ADOS- : Autism Diagnostic Observation Schedule, Generic; K: thousand.

\section{Measures}

The Autism Quotient (AQ; Baron-Cohen et al., 2001) is a 50-item measure of autism features. In this study, autistic adults completed the self-report version for ages 16 and older. Each item on the questionnaire provides four response options: definitely agree, slightly agree, slightly disagree, and definitely disagree. A sample item is, "I frequently find that I don't know how to keep a conversation going." Consistent with previous research, responses were coded on a 4-point scale (Murray et al., 2017) and summed to create a Total score, with higher scores indicating more autistic features. The $A Q$ has been shown to have good test-retest reliability and acceptable internal consistency (Baron-Cohen et al., 2001). Support for the originally established 5-factor structure (Social Skill, Attention Switching, Attention to Detail, Communication, and Imagination) has been weak in the literature (Kloosterman et al., 2011), with one study noting that associations between specific subscales of the $A Q$ and other variables are confounded by a more general factor (Murray et al., 2017). Based on these studies, only the Total score was used in the present analyses. Internal consistency here was acceptable for the Total score $(\alpha=0.77)$.

The Friendship Questionnaire (FQ; Baron-Cohen \& Wheelwright, 2003) is a 35-item questionnaire designed for use with both neurotypical and autistic adults. The item "How often do you make plans to meet with friends?" was used in this study as a proxy of initiated social contact for sake of measurement specificity, rather than the multiple aspects of friendship that the $\mathrm{FQ}$ are intended to measure (e.g. friendship knowledge, social motivation, friendship quality, social skills). Response options ranged from 0 (once or twice a year) to 5 (3 or 4 times a week or more).

The Social and Emotional Loneliness Scale for Adults (SELSA; DiTommaso \& Spinner, 1993) is a measure of loneliness. The questionnaire consists of 37 items, 14 of which comprise the Social Loneliness subscale (SOC), along with 11 in the Emotional Family Loneliness (EFAM) subscale and 12 in the Emotional Romantic Loneliness subscale (EROM). Item responses are on a 7-point Likert-type scale from 1 (strongly disagree) to 7 (strongly agree) on statements such as "I'm not part of a group of friends, and I wish I were." Higher scores indicate greater loneliness. The SELSA has demonstrated good convergent validity with the UCLA Loneliness Scale (DiTommaso \& Spinner, 1993) and has been previously used in studies of autistic adults (Gantman et al., 2012; McVey et al., 2016). Here, internal consistency was excellent for the SOC subscale $(\alpha=0.93)$, good for the EFAM subscale $(\alpha=0.89)$, and acceptable for the EROM subscale $(\alpha=0.73)$.

Two measures of social anxiety were used, following the recommendations of a recent systematic review of social anxiety in autism (Spain et al., 2018). The Social Phobia Inventory (SPIN; Connor et al., 2000) is a 17-item questionnaire assessing social anxiety symptoms during the previous week. It uses a 5-point Likert-type from 0 (not at all) to 4 (extremely). A sample item is "Talking to strangers scares me." The SPIN has demonstrated high reliability and acceptable convergent validity (Connor et al., 2000). The SPIN has been used in previous studies of adults on the autism spectrum (e.g. Langdon et al., 2016). The internal consistency in this study was excellent $(\alpha=0.93)$. The self-report version of the Leibowitz Social Anxiety Scale (LSAS; Baker et al., 2002; Fresco et al., 2001) consists of 24 items inquiring about a range of social situations. Each item contains a brief description of a social situation, for example, "Meeting strangers (assume other are of average importance to you)" and asks for ratings of both anxiety and avoidance. Adults indicate on a 4-point Likert-type scale from 0 (none) to 3 (severe) the intensity of their anxiety in that situation over the past week and how much of the time, from 0 (never $0 \%$ ) to 3 (usually $67 \%-100 \%$ ), they have avoided that situation. Higher scores indicate greater social anxiety. The measure has shown acceptable internal consistency and convergent validity for the original clinician- 
administered and self-report versions (Fresco et al., 2001; Heimberg et al., 1999) in neurotypical samples, and it has been used to measure social anxiety in research on autism (Perry et al., 2015). Internal consistency in this study was excellent $(\alpha=0.96)$.

The Beck Depression Inventory, Second Edition (BDI-II; Beck et al., 1996) is a 21-item self-report questionnaire that assesses depressive symptoms in people ages 13-80. Respondents use a Likert-type rating scale to select one of four statements per item. A sample group of statements is, "I do not feel sad; I feel sad much of the time; I am sad all the time; I am so sad or unhappy that I can't stand it." The BDI-II boasts good psychometric properties including internal consistency, concurrent validity, and test-retest reliability (Dozois et al., 1998; Sprinkle et al., 2002; Storch et al., 2004). The Total score is a sum of all item scores, with higher scores indicating greater levels of depressive symptoms. The BDI-II has been identified as a reasonable instrument for measuring depression in adults on the autism spectrum (Cassidy et al., 2018; Gotham et al., 2015). Internal consistency here was excellent $(\alpha=0.92)$.

\section{Data analytic plan}

All analyses were run in R version 3.5.0 (R Core Team, 2013). Data were screened for outliers, and internal reliability for each measure was calculated using the Procedures for Psychological, Psychometric, and Personality Research (Psych; Revelle, 2018) package. Descriptive statistics and Pearson's correlations were calculated using the Psych package (Revelle, 2018) and Harrell Miscellaneous (Hmisc; Harrell et al., 2019) package, respectively.

Mediation models were run using the latent variable analysis (lavaan; Rosseel, 2012) package. Our analyses tested the direct and indirect effects from autism features (AQ) and number of social contacts (FQ) to depression (BDI-II) and social anxiety (LSAS, SPIN) through social loneliness (SELSA). For visual depiction, see Figure 1. Bootstrapped confidence intervals (Cls) were used to estimate indirect effects, as this approach is particularly well-suited for small samples. MedPower was used to determine a sample size of 63 was necessary to achieve adequate power $(B=0.80)$ to detect indirect effects at an alpha level of 0.05 (Kenny, 2017). Models were run three times utilizing the SELSA SOC subscale, the EFAM subscale, and then the EROM subscale.

\section{Results}

\section{Correlations and preliminary analyses}

A full correlation matrix is presented, including all outcome variables and demographic characteristics, in Table 2. Bivariate Pearson's correlations indicated that autism features (AQ Total score) were significantly positively associated with social loneliness (SELSA SOC: $r=0.49, p<0.01$ ), family loneliness (SELSA

EFAM: $r=0.41, p<0.01$ ), social anxiety (LSAS: $r=0.54, p<0.01$; SPIN: $r=0.33, p<0.01$ ), and depression (BDIII: $r=0.34, p=0.02$ ) and significantly negatively associated with number of initiated social contacts (FQ: $r=-0.29, p=0.05$ ). No relation was uncovered with romantic loneliness (SELSA EROM). That is, autistic adults who reported more autism features were likely to report more social loneliness, higher levels of social anxiety and depression, and fewer initiated social contacts. 
Table 2. Bivariate correlations among study variables.

\begin{tabular}{|c|c|c|c|c|c|c|c|c|c|c|c|}
\hline & $M$ & SD & 1. & 2. & 3. & 4. & 5. & 6. & 7. & 8. & 9. \\
\hline 1. AQ Total & 128.01 & 13.77 & - & & & & & & & & \\
\hline 2. FQ SC & 2.24 & 1.80 & $-0.29 *$ & - & & & & & & & \\
\hline 3. SELSA SOC & 47.26 & 21.13 & $0.49 * *$ & $-0.53 * *$ & - & & & & & & \\
\hline 4. SELSA EFAM & 25.35 & 12.81 & $0.41^{* *}$ & $-0.27^{*}$ & $0.45^{* *}$ & - & & & & & \\
\hline 5. SELSA EROM & 56.19 & 13.19 & 0.23 & -0.17 & $0.37^{* *}$ & $0.31^{*}$ & & & & & \\
\hline 6. SPIN & 28.06 & 16.14 & $0.33 * *$ & $-0.35^{* *}$ & $0.52 * *$ & $0.40 * *$ & 0.22 & - & & & \\
\hline 7. LSAS & 57.35 & 30.21 & $0.54 * *$ & $-0.42 * *$ & $0.59 * *$ & $0.47^{* *}$ & $0.25 *$ & $0.74 * *$ & - & & \\
\hline 8. BDI-II & 12.58 & 10.99 & $0.34^{* *}$ & -0.15 & $0.44^{* *}$ & $0.72 * *$ & $0.31^{*}$ & $0.43^{* *}$ & $0.48^{* *}$ & - & \\
\hline 9. Age & 20.16 & 2.97 & 0.12 & 0.01 & -0.01 & -0.01 & 0.04 & -0.10 & 0.03 & -0.02 & - \\
\hline 10. KBIT-2 IQ & 95.01 & 17.43 & $0.24^{*}$ & -0.18 & 0.18 & 0.04 & 0.21 & 0.04 & 0.18 & 0.01 & -0.08 \\
\hline
\end{tabular}

M: mean; SD: standard deviation; AQ: Autism Quotient; FQ SC: Friendship Questionnaire Social Contact; SELSA: Social and Emotional Loneliness Scale for Adults; SOC: Social Loneliness subscale; EFAM: Emotional Family Loneliness subscale; EROM: Emotional Romantic Loneliness subscale; SPIN: Social

Phobia Inventory; LSAS: Leibowitz Social Anxiety Scale; BDI-II: Beck Depression Inventory, Second Edition; KBIT-2: Kaufman Brief Intelligence Test, Second Edition.

${ }^{*} p<0.05 ;{ }^{* *} p<$ 
Number of social contacts demonstrated significant negative associations with social loneliness (SELSA SOC: $r=-0.53, p<0.01$ ), family loneliness (SELSA EFAM: $r=-0.27, p<0.05$ ), and social anxiety

(LSAS: $r=-0.42, p=<0.01$; SPIN: $r=-0.35, p<0.01$ ). No association was identified with romantic loneliness (EROM) or depression (BDI-II). Thus, our findings revealed that autistic adults who indicated more social contact initiations were likely to report lower levels of social loneliness and social anxiety but not depression.

Exploring associations between the outcome variables with age and IQ revealed a significant positive association between autism features (AQ) and IQ $(r=0.24, p<0.05)$. No relations were observed with age. Independent samples $t$ tests indicated that none of the outcome variables differed across gender. Therefore, we ran all mediation models both with and without IQ as a covariate. Results indicated identical interpretation of parameter estimates for models with and without IQ as a covariate, and thus, for sake of parsimony, results are presented from only the set of models excluding IQ.

\section{Mediation models}

Results for our mediation model predicting social anxiety on the SPIN are depicted in Figure 2 . Results indicated nonsignificant direct effects of number of social contacts (FQ: $B=-0.11, B=-1.00, p=0.42$ ) and autism features ( $A Q: B=0.07, B=0.09, p=0.57$ ) on social anxiety (SPIN), controlling for social loneliness (SELSA SOC). Social loneliness (SELSA SOC) was a significant predictor of social anxiety (SPIN: $B=0.41 ; B=0.31, p=0.01$ ) controlling for the effects of autism features (AQ) and social contacts (FQ). This suggests that, accounting for differences in levels of autism features ( $\mathrm{AQ}$ ) and social contacts (FQ), greater social loneliness was related to more social anxiety. Analyses also indicated that social contacts (FQ: $B=-0.42 ; B=-4.90 p<0.01$ ) and autism traits (AQ: $B=0.39 ; B=0.62 p<0.01$ ) each explained unique variance in social loneliness (SELSA SOC). Less frequent social contact and more autism features were each uniquely predictive of greater social loneliness.

Bootstrapped Cls indicated that the indirect effect of autism traits (AQ) on social anxiety (SPIN) through social loneliness (SELSA SOC) was significant $(B=0.19, \mathrm{Cl}: 0.05,0.43)$, and the indirect effect of social contacts (FQ) on social anxiety (SPIN) through social loneliness (SELSA SOC) was also significant $(B=-1.52, \mathrm{Cl}:-3.01,-0.38)$. The nonsignificant direct effects (described above) provide evidence for full mediation.

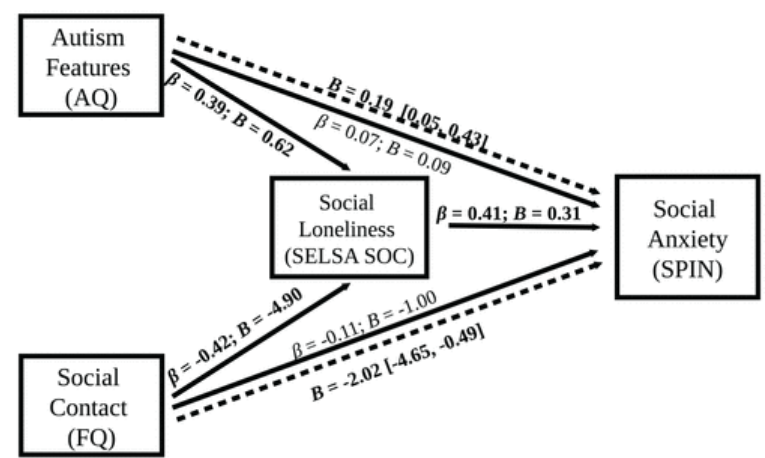

Figure 2. Results for mediation model with SPIN social anxiety measure.

Note. Solid lines indicate direct effects. Dotted lines indicate indirect effects. $B=$ unstandardized parameter. $B=$ standardized parameter. Bolded values are significant at alpha level of 0.05 .

With one exception, our pattern of results using the LSAS in place of the SPIN as a measure of social anxiety remained identical (Figure 3). The only difference between the models was a significant direct effect of autism features (AQ: $B=0.31 ; B=0.70, p<0.01$ ) on social anxiety (LSAS). Thus, holding social loneliness (SELSA SOC) and social contacts (FQ) constant, more autism features was associated with greater social anxiety. Therefore, there was only evidence for partial mediation of autism features (AQ) on social anxiety (LSAS) through social loneliness (SELSA SOC) using the LSAS. Otherwise, interpretation of all parameter estimates for direct and indirect effects remained consistent across both social anxiety measures. 


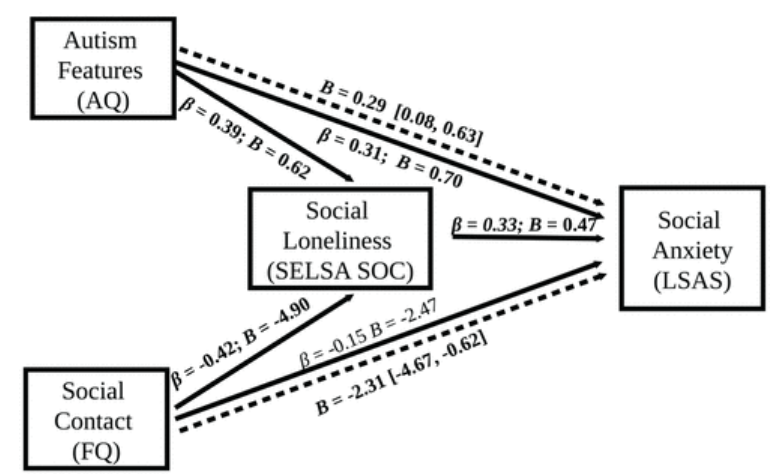

Figure 3. Results for mediation model with LSAS social anxiety measure.

Note. Solid lines indicate direct effects. Dotted lines indicate indirect effects. $B=$ unstandardized parameter. $B=$ standardized parameter. Bolded values are significant at alpha level of 0.05 .

For mediation models predicting depression, we found an identical pattern for depression parameters as identified for predicting the SPIN (Figure 4). In brief, the indirect effects of number of social contacts (FQ) and autism features (AQ) on depression (BDI-II) through social loneliness (SELSA SOC) were significant (FQ: $B=-1.52$, $\mathrm{Cl}$ :-3.01, -0.38; $\mathrm{AQ:} B=0.19, \mathrm{Cl}: 0.05,0.42$ ). Direct effects from social contacts (FQ) and autism features (AQ) on depression (BDI-II) were nonsignificant, providing evidence of full mediation.

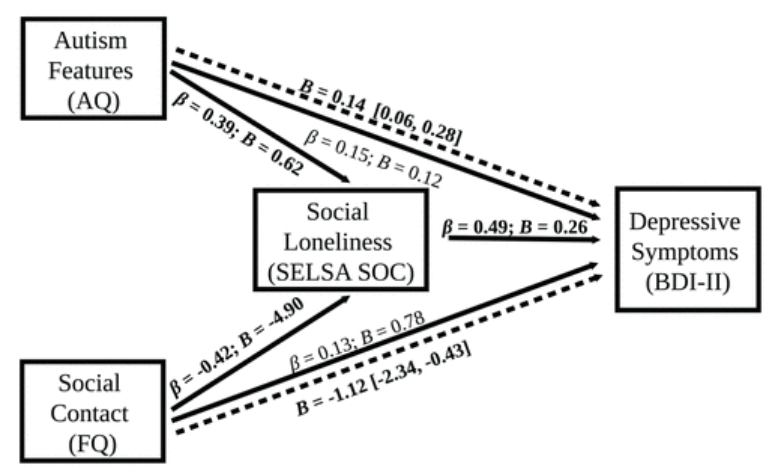

Figure 4. Results for mediation model with BDI-II depression measure.

Note. Solid lines indicate direct effects. Dotted lines indicate indirect effects. $B=$ unstandardized parameter. $B=$ standardized parameter. Bolded values are significant at alpha level of 0.05 .

All models were re-run substituting the SELSA EFAM and EROM subscales for the SOC subscale (Tables 3 and 4). Models using the EFAM subscale partially held (Table 3). In particular, the indirect effect of autism features (AQ) through family loneliness (EFAM) to mental health was significant. For one of the social anxiety measures (SPIN) and depressive symptoms (BDI-II), there was evidence for full mediation, while there was only evidence for partial mediation for the other social anxiety measure (LSAS). None of the identified indirect effects held using the EROM subscale (Table 4). 
Table 3. Mediation models predicting mental health outcomes using SELSA EFAM subscale.

\begin{tabular}{|c|c|c|c|c|c|c|c|}
\hline $\begin{array}{l}\text { Mental health } \\
\text { outcome }\end{array}$ & $\begin{array}{l}\text { Direct } \\
\text { effects }\end{array}$ & & & & & Indirect effects & \\
\hline & a1 & a2 & $b$ & $c 1^{\prime}$ & $c 2^{\prime}$ & $a 1 * b$ & $a 2 * b$ \\
\hline & $\begin{array}{l}\text { AQ to } \\
\text { SELSA }\end{array}$ & FQ to SELSA & $\begin{array}{l}\text { SELSA to } \\
\text { mental health }\end{array}$ & $\begin{array}{l}\text { AQ to mental } \\
\text { health }\end{array}$ & $\begin{array}{l}\text { FQto mental } \\
\text { health }\end{array}$ & $\begin{array}{l}\text { AQ to mental health } \\
\text { through SELSA }\end{array}$ & $\begin{array}{l}\text { FQ to mental health } \\
\text { through SELSA }\end{array}$ \\
\hline \multicolumn{8}{|l|}{ Anxiety } \\
\hline SPIN & $0.37(0.16)$ & $-1.13(0.95)$ & $0.39(0.16)$ & $0.13(0.13)$ & $-2.08(1.16)$ & $0.15[0.03,0.34]$ & $-0.44[-1.65,0.22]$ \\
\hline LSAS & $0.38(0.16)$ & $-1.13(0.89)$ & $0.64(0.22)$ & $0.74(0.21)$ & $-4.05(1.71)$ & $0.24[0.05,0.55]$ & $-0.73[-2.63,0.23]$ \\
\hline \multicolumn{8}{|l|}{ Depression } \\
\hline BDI-II & $0.38(0.15)$ & $-1.13(0.89)$ & $0.60(0.11)$ & $0.04(0.10)$ & $0.33(0.57)$ & $0.22[0.05,0.45]$ & $-0.68[-2.09,0.24]$ \\
\hline
\end{tabular}

SELSA: Social and Emotional Loneliness Scale for Adults; EFAM: Emotional Family Loneliness subscale; AQ: Autism Quotient; FQ: Friendship Questionnaire Social Contact; SPIN: Social Phobia Inventor ; LSAS: Leibowitz Social Anxiety Scale; BDI-II: Beck Depression Inventory, Second Edition. Bolded parameters are significant at an alpha level of 0.05

Table 4. Mediation models predicting mental health outcomes using SELSA EROM subscale.

\begin{tabular}{|c|c|c|c|c|c|c|c|}
\hline $\begin{array}{l}\text { Mental } \\
\text { health }\end{array}$ & $\begin{array}{l}\text { Direct } \\
\text { effects }\end{array}$ & & & & & Indirect effects & \\
\hline & a1 & a2 & $b$ & c1' & c2` & $a 1 * b$ & $a 2 * b$ \\
\hline & $\begin{array}{l}\text { AQ to } \\
\text { SELSA }\end{array}$ & FQ to SELSA & $\begin{array}{l}\text { SELSA to } \\
\text { mental health }\end{array}$ & $\begin{array}{l}A Q \text { to mental } \\
\text { health }\end{array}$ & $\begin{array}{l}\text { FQ to Mental } \\
\text { Health }\end{array}$ & $\begin{array}{l}\text { AQ to mental health } \\
\text { through SELSA }\end{array}$ & $\begin{array}{l}\text { FQ to mental health } \\
\text { through SELSA }\end{array}$ \\
\hline \multicolumn{8}{|l|}{ Anxiety } \\
\hline SPIN & $0.13(0.16)$ & $-1.01(1.11)$ & $0.18(0.16)$ & $0.26(0.13)$ & $-2.34(1.16)$ & $0.02[-0.03,0.16]$ & $-0.18[-1.34,0.10]$ \\
\hline LSAS & $0.13(0.15)$ & $-1.01(1.09)$ & $0.17(0.24)$ & $0.96(0.21)$ & $-4.61(1.79)$ & $0.02[-0.03,0.28]$ & $-0.18[-1.67,0.22]$ \\
\hline \multicolumn{8}{|c|}{ Depression } \\
\hline BDI-II & $0.13(0.13)$ & $-1.01(1.05)$ & $0.21(0.10)$ & $0.23(0.11)$ & $-0.13(0.69)$ & $0.03[-0.03,3.18]$ & $-0.22[-0.99,0.15]$ \\
\hline
\end{tabular}

SELSA: Social and Emotional Loneliness Scale for Adults; EROML Emotional Romantic Loneliness Subscale; AQ: Autism Quotient; FQ: Friendship Questionnaire Social Contact; SPIN: Social Phobia Inventory; LSAS: Leibowitz Social Anxiety Scale; BDI-II: Beck Depression Inventory, Second Edition. Bolded parameters are significant at an alpha level of 0.05 . 


\section{Discussion}

This study's findings indicate that loneliness plays an important role in social anxiety and depression among autistic young adults. These results extend previous research that identified associations between autism features and mental health concerns among autistic people (Factor et al., 2017; Mayes et al., 2011; McVey et al., 2018) by identifying an indirect effect through loneliness. Similar effects have been identified in a non-clinical, neurotypical college student sample (Stice \& Lavner, 2019), suggesting that parallel processes may be at play for autistic adults.

Furthermore, these findings add to the burgeoning collection of studies focused upon loneliness and mental health in autism. A recent study showed higher levels of loneliness reported by autistic adults compared to nonautistic adults (Ee et al., 2019), suggesting that loneliness is particularly salient for this population. Among autistic adults, number of friends and loneliness have each been found to explain unique variance in depression and anxiety (Mazurek, 2014). Based on our analyses, number of initiated social contacts and social loneliness did not independently predict mental health concerns-instead, social loneliness mediated the effect of social contact. These divergent findings may relate to the differing aspects of friendship that were measured (i.e. quantity of friends vs number of initiated social contacts). In addition, our analyses revealed that variance in social loneliness was explained by a combination of more autism features and less social contact. While these two factors were correlated in our sample, they contributed independently to loneliness. These findings are commensurate with those of Ee and colleagues (2019) who identified both social support and social skills as unique predictors of loneliness among autistic adults. As such, the experience of loneliness for adults on the autism spectrum seems to be related to direct social interaction as well as perceived level of social competency.

A novel aspect of this study is the various types of loneliness that were explored among autistic adults. The differential effects for social versus emotional loneliness are noteworthy. That is, the indirect effects described above for social loneliness did not hold using romantic loneliness (i.e. emotional loneliness related to romantic relationships) and only partially held using family loneliness (i.e. emotional loneliness related to family relationships). Although previous work indicates that many autistic adults desire romantic relationships (Strunz et al., 2017), these findings suggest that, for autistic young adults, a lack of friendships may play a larger role in mental health than emotional romantic connections. Interestingly, family loneliness was related to autistic features and mediated the link to mental health concerns. Consistent with prior work (e.g. Beurkens et al., 2013), it may be that even within families, bonds with parents, siblings, and extended family are influenced by social challenges inherent in autism; this, in turn, may result in feelings of isolation and poorer mental health.

Clinically, autistic youth and young adults who receive a social skills intervention have been shown to demonstrate secondary improvements in social anxiety and depression (Corbett et al., 2017; Lei et al., 2017; McVey et al., 2016; Schiltz et al., 2018; Schohl et al., 2014; Yoo et al., 2014), yet the mechanisms of this effect are not yet well understood. Our findings here point to loneliness as one important factor that may explain these secondary improvements in mental health. As autistic youth and young adults learn and practice ecologically valid social skills, they increase the number of successful social interactions, experience less loneliness, and demonstrate fewer symptoms of social anxiety and depression. Therefore, social skills interventions seem to have crucial effects on the mental well-being of autistic adults that extend beyond mere social contact, and which may occur through reduced feelings of loneliness. Clinical implications of these findings point to the importance of mental health providers assessing and targeting social relationships, perhaps in order to ameliorate feelings of loneliness, when autistic clients present for treatment of anxiety and depression. When possible, referral to evidence-based social skills interventions may especially be helpful. Anxiety and depression in autism are associated with an increased risk for suicidality (Hooijer \& Sizoo, 2019), and recent findings reveal concerningly high rates of suicidality among people on the autism spectrum (Cassidy 
et al., 2014; Griffiths et al., 2019; Hirvikoski et al., 2020), highlighting the importance of preventive efforts. The current findings point to addressing loneliness as one avenue toward promoting resilience and mental wellbeing and reducing suicidality among autistic adults.

Despite these important findings, our study had several limitations that provide direction for future research. First, our cross-sectional design limits our ability to draw causal conclusions. In contrast to our discussion above, it may be that mental health concerns increase loneliness, or that these factors have a bidirectional association across time. That is, loneliness may be a consequence of heightened social isolation, avoidance, or withdrawal due to depression or social anxiety. Future studies should aim to use a longitudinal methodology to test directional effects of these pathways. Second, the sample was limited. Because the autistic young adults were seeking participation in a social skills intervention, it might be that loneliness, social anxiety, and depressive symptoms were more prevalent in this sample than the general autistic community. The sample was also relatively sociodemographic homogeneous (i.e. race, ethnicity, verbal ability). These factors limit generalization of our results. Third, because our measures of loneliness (SELSA) and social anxiety (LSAS, SPIN) have not received robust psychometric evaluation in an autistic sample, we do not know whether these tools reliably measure the intended constructs in autism. It is noteworthy, however, that both measures of social anxiety (LSAS, SPIN) functioned similarly and yielded nearly identical results, pointing to consistency of measurement. Fourth, given the poor support for use of subscales on the $A Q$, we were not able to test which aspects of autism features may be driving the identified effects. Future studies with very large samples may wish to replicate these findings using a bifactor model to explore the effects of subscales while removing variance due to the general factor (Murray et al., 2017). Fifth, all measures in this study were self-report, and therefore other metrics including reports of caregivers, clinician interviews, and qualitative data would be beneficial to explore these processes from multiple informants and perspectives. Qualitative exploration, in particular, of social contact, loneliness, and quality of relationships would provide richer information about the lived experiences of autistic adults (for instance, see Sedgewick et al., 2019). Finally, a range of factors not measured here should continue to be considered in future research on pathways between autism features and mental health. These include stigma and social rejection, as autistic adults with more pronounced autism features face greater social rejection (Rotheram-Fuller et al., 2010; Sebastian et al., 2009), as well as unemployment and self-efficacy, as negative experiences related to employment and education have been related to mental health in autistic adults (e.g. Griffiths et al., 2019).

In conclusion, this study found evidence for loneliness as a mediator explaining the connections between autism features and social contact with social anxiety and depression among autistic adults. Identifying loneliness as a potential root of these common mental health concerns helps to better clarify the needs of autistic young adults - an especially vulnerable population, given the unique needs of this developmental stage. These findings help to explain prior research that identified improvements in social anxiety and depression across social skills interventions. The results of this study have significant implications for research in elucidating processes of mental health concerns among autistic adults as well as clinical practice in assessing and targeting loneliness in intervention for autistic adults.

\section{Acknowledgements}

The authors would like to thank the autistic young adults and their families who participated in the study. The authors would also like to recognize the entire Marquette Autism Project research team without whose efforts this work would not be possible. An earlier iteration of this project was presented as a poster at the International Society for Autism Research 2019 Annual Meeting under the title, "Loneliness Mediates the Association between Friendship, Autism Symptoms, and Mental Health in Young Adults with Autism." 


\section{Author's Note}

Alana J McVey is now affiliated to The University of British Columbia, Vancouver, BC, Canada.

\section{Funding}

The author(s) disclosed receipt of the following financial support for the research, authorship, and/or publication of this article: The authors would also to recognize the Autism Society of Southeastern Wisconsin for their funding support of the larger randomized clinical trial of the PEERS ${ }^{\bullet}$ interventions. The first author of this work would also like to acknowledge the funding support of the Richard W. Jobling Distinguished Research Assistantship at Marquette University.

\section{References}

American Psychiatric Association . (2013). Diagnostic and statistical manual of mental disorders (DSM-5 ${ }^{\circledR}$ ) (5th ed.). American Psychiatric Publishing.

Baker, S. L., Heinrichs, N., Kim, H.-J., Hofmann, S. G. (2002). The Liebowitz social anxiety scale as a self-report instrument: A preliminary psychometric analysis. Behaviour Research and Therapy, 40(6), 701-715.

Bandelow, B., Michaelis, S. (2015). Epidemiology of anxiety disorders in the 21st century. Dialogues in Clinical Neuroscience, 17(3), 327-335. https://www.ncbi.nlm.nih.gov/pubmed/26487813

Baron-Cohen, S., Wheelwright, S. (2003). The Friendship Questionnaire: An investigation of adults with Asperger syndrome or high-functioning autism, and normal sex differences. Journal of Autism and Developmental Disorders, 33(5), 509-517.

Baron-Cohen, S., Wheelwright, S., Skinner, R., Martin, J., Clubley, E. (2001). The autism-spectrum quotient (AQ): Evidence from Asperger syndrome/high-functioning autism, males and females, scientists and mathematicians. Journal of Autism and Developmental Disorders, 31(1), 5-17.

Bauminger, N., Kasari, C. (2000). Loneliness and friendship in high-functioning children with autism. Child Development, 71(2), 447-456.

Beck, A. T., Steer, R. A., Brown, G. K. (1996). Beck Depression Inventory. Psychological Corporation.

Bejerot, S., Eriksson, J. M., Mörtberg, E. (2014). Social anxiety in adult autism spectrum disorder. Psychiatry Research, 220(1), 705-707.

Bellini, S. (2006). The development of social anxiety in adolescents with autism spectrum disorders. Focus on Autism and Other Developmental Disabilities, 21(3), 138-145.

Beurkens, N. M., Hobson, J. A., Hobson, R. P. (2013). Autism severity and qualities of parent-child relations. Journal of Autism and Developmental Disorders, 43(1), 168-178.

Cassidy, S. A., Bradley, L., Bowen, E., Wigham, S., Rodgers, J. (2018). Measurement properties of tools used to assess depression in adults with and without autism spectrum conditions: A systematic review. Autism Research, 11(5), 738-754.

Cassidy, S. A., Bradley, P., Robinson, J., Allison, C., McHugh, M., Baron-Cohen, S. (2014). Suicidal ideation and suicide plans or attempts in adults with Asperger's syndrome attending a specialist diagnostic clinic: A clinical cohort study. The Lancet Psychiatry, 1(2), 142-147.

Connor, K. M., Davidson, J. R. T., Churchill, L. E., Sherwood, A., Weisler, R. H., Foa, E. (2000). Psychometric properties of the Social Phobia Inventory (SPIN): New self-rating scale. The British Journal of Psychiatry, 176(4), 379-386.

Corbett, B. A., Blain, S. D., loannou, S., Balser, M. (2017). Changes in anxiety following a randomized control trial of a theatre-based intervention for youth with autism spectrum disorder. Autism, 21(3), 333-343.

DiTommaso, E., Spinner, B. (1993). The development and initial validation of the Social and Emotional Loneliness Scale for Adults (SELSA). Personality and Individual Differences, 14(1), 127-134.

Dozois, D. J. A., Dobson, K. S., Ahnberg, J. L. (1998). A psychometric evaluation of the Beck Depression Inventory-II. Psychological Assessment, 10(2), 83-89. 
Ee, D., Hwang, Y. I., Reppermund, S., Srasuebkul, P., Trollor, J. N., Foley, K.-R., Arnold, S. R. C. (2019). Loneliness in adults on the autism spectrum. Autism in Adulthood, 1(3), 182-193.

Factor, R. S., Ryan, S. M., Farley, J. P., Ollendick, T. H., Scarpa, A. (2017). Does the presence of anxiety and ADHD symptoms add to social impairment in children with autism spectrum disorder? Journal of Autism and Developmental Disorders, 47(4), 1122-1134.

Fresco, D. M., Coles, M. E., Heimberg, R. G., Liebowitz, M. R., Hami, S., Stein, M. B., Goetz, D. (2001). The Liebowitz Social Anxiety Scale: A comparison of the psychometric properties of self-report and clinician-administered formats. Psychological Medicine, 31(6), 1025-1035.

Gantman, A., Kapp, S. K., Orenski, K., Laugeson, E. A. (2012). Social skills training for young adults with highfunctioning autism spectrum disorders: A randomized controlled pilot study. Journal of Autism and Developmental Disorders, 42(6), 1094-1103.

Gotham, K., Unruh, K., Lord, C. (2015). Depression and its measurement in verbal adolescents and adults with autism spectrum disorder. Autism, 19(4), 491-504.

Griffiths, S., Allison, C., Kenny, R., Holt, R., Smith, P., Baron-Cohen, S. (2019). The Vulnerability Experiences Quotient (VEQ): A study of vulnerability, mental health and life satisfaction in autistic adults. Autism Research, 12(10), 1516-1528.

Harrell, F. E., Dupont, C., \& many others. (2019). Hmisc: Harrell miscellaneous (R Package Version $4.2-$ 0). https://CRAN.R-project.org/package=Hmisc

Hasin, D. S., Sarvet, A. L., Meyers, J. L., Saha, T. D., Ruan, W. J., Stohl, M., Grant, B. F. (2018). Epidemiology of adult DSM-5 major depressive disorder and its specifiers in the United States. JAMA Psychiatry, 75(4), 336-346. https://doi.org/10.1001/jamapsychiatry.2017.4602

Hedley, D., Uljarević, M., Wilmot, M., Richdale, A., Dissanayake, C. (2018). Understanding depression and thoughts of self-harm in autism: A potential mechanism involving loneliness. Research in Autism Spectrum Disorders, 46, 1-7.

Hedley, D., Young, R. (2006). Social comparison processes and depressive symptoms in children and adolescents with Asperger syndrome. Autism, 10(2), 139-153.

Heimberg, R. G., Horner, K. J., Juster, H. R., Safren, S. A., Brown, E. J., Schneier, F. R., Liebowitz, M. R. (1999). Psychometric properties of the Liebowitz Social Anxiety Scale. Psychological Medicine, 29(1), 199-212.

Hirvikoski, T., Boman, M., Chen, Q., D’Onofrio, B. M., Mittendorfer-Rutz, E., Lichtenstein, P., Bölte, S., Larsson, H. (2020). Individual risk and familial liability for suicide attempt and suicide in autism: A populationbased study. Psychological Medicine, 50(9), 1463-1474.

Hofvander, B., Delorme, R., Chaste, P., Nydén, A., Wentz, E., Ståhlberg, O., Herbrecht, E., Stopin, A., Anckarsäter, H., Gillberg, C. (2009). Psychiatric and psychosocial problems in adults with normalintelligence autism spectrum disorders. BMC Psychiatry, 9(1), Article 35.

Hollocks, M. J., Lerh, J. W., Magiati, I., Meiser-Stedman, R., Brugha, T. S. (2019). Anxiety and depression in adults with autism spectrum disorder: A systematic review and meta-analysis. Psychological Medicine, 49(4), 559-572.

Hong, J., Bishop-Fitzpatrick, L., Smith, L. E., Greenberg, J. S., Mailick, M. R. (2016). Factors associated with subjective quality of life of adults with autism spectrum disorder: Self-report versus maternal reports. Journal of Autism and Developmental Disorders, 46(4), 1368-1378.

Hooijer, A. A. T., Sizoo, B. B. (2019). Temperament and character as risk factor for suicide ideation and attempts in adults with autism spectrum disorders. Autism Research, 13(1), 104-111.

Kanne, S. M., Christ, S. E., Reiersen, A. M. (2009). Psychiatric symptoms and psychosocial difficulties in young adults with autistic traits. Journal of Autism and Developmental Disorders, 39(6), 827-833.

Kapp, S. K. (2018). Social support, well-being, and quality of life among individuals on the autism spectrum. Pediatrics, 141(Suppl. 4), S362-S368.

Kaufman, A. S., Kaufman, N. L. (2004). Kaufman Brief Intelligence Test, second edition (KBIT-2). Pearson.

Kenny, D. A. (2017). MedPower: An interactive tool for the estimation of power in tests of mediation. https://davidakenny.shinyapps.io/MedPower/ 
Kloosterman, P. H., Keefer, K. V., Kelley, E. A., Summerfeldt, L. J., Parker, J. D. A. (2011). Evaluation of the factor structure of the autism-spectrum quotient. Personality and Individual Differences, 50(2), 310314.

Langdon, P. E., Murphy, G. H., Shepstone, L., Wilson, E. C. F., Fowler, D., Heavens, D., Russell, A., Rose, A., Malovic, A., Mullineaux, L. (2016). The people with Asperger syndrome and anxiety disorders (PAsSA) trial: A pilot multicentre, single-blind randomised trial of group cognitive-behavioural therapy. BJPsych Open, 2(2), 179-186.

Laugeson, E. A. (2017). PEERS ${ }^{\circledast}$ for young adults: Social skills training for adults with autism spectrum disorder and other social challenges. Routledge.

Lei, J., Sukhodolsky, D. G., Abdullahi, S. M., Braconnier, M. L., Ventola, P. (2017). Reduced anxiety following pivotal response treatment in young children with autism spectrum disorder. Research in Autism Spectrum Disorders, 43, 1-7.

Locke, J., Ishijima, E. H., Kasari, C., London, N. (2010). Loneliness, friendship quality and the social networks of adolescents with high-functioning autism in an inclusive school setting. Journal of Research in Special Educational Needs, 10(2), 74-81.

Lord, C., Rutter, M., DiLavore, P. C., Risi, S., Gotham, K., Bishop, S. L. (2001). Autism Diagnostic Observation Schedule (ADOS). Western Psychological Services.

Maddox, B. B., White, S. W. (2015). Comorbid social anxiety disorder in adults with autism spectrum disorder. Journal of Autism and Developmental Disorders, 45(12), 3949-3960.

Mayes, S. D., Calhoun, S. L., Murray, M. J., Zahid, J. (2011). Variables associated with anxiety and depression in children with autism. Journal of Developmental and Physical Disabilities, 23(4), 325-337.

Mazurek, M. O. (2014). Loneliness, friendship, and well-being in adults with autism spectrum disorders. Autism, 18(3), 223-232.

Mazurek, M. O., Kanne, S. M. (2010). Friendship and internalizing symptoms among children and adolescents with ASD. Journal of Autism and Developmental Disorders, 40(12), 1512-1520.

McVey, A. J., Dolan, B. K., Willar, K. S., Pleiss, S., Karst, J. S., Casnar, C. L., Caiozzo, C., Vogt, E. M., Gordon, N. S., Van Hecke, A. V. (2016). A replication and extension of the PEERS ${ }^{\circledR}$ for young adults social skills intervention: Examining effects on social skills and social anxiety in young adults with autism spectrum disorder. Journal of Autism and Developmental Disorders, 46(12), 3739-3754.

McVey, A. J., Schiltz, H. K., Haendel, A. D., Dolan, B. K., Willar, K. S., Pleiss, S. S., Karst, J. S., Carlson, M., Krueger, W., Murphy, C. C. (2018). Social difficulties in youth with autism with and without anxiety and ADHD symptoms. Autism Research, 11(12), 1679-1689.

Morton, H. E., Gillis, J. M., Mattson, R. E., Romanczyk, R. G. (2019). Conceptualizing bullying in children with autism spectrum disorder: Using a mixed model to differentiate behavior types and identify predictors. Autism, 23(7), 1853-1864.

Murray, A. L., McKenzie, K., Kuenssberg, R., Booth, T. (2017). Do the autism spectrum quotient (AQ) and autism spectrum quotient short form (AQ-S) primarily reflect general ASD traits or specific ASD traits? A bi-factor analysis. Assessment, 24(4), 444-457.

O'Hagan, S., Hebron, J. (2017). Perceptions of friendship among adolescents with autism spectrum conditions in a mainstream high school resource provision. European Journal of Special Needs Education, 32(3), 314-328.

Peplau, L. A. (1982). Loneliness: A sourcebook of current theory, research, and therapy (Vol. 36). John Wiley.

Perry, A., Levy-Gigi, E., Richter-Levin, G., Shamay-Tsoory, S. G. (2015). Interpersonal distance and social anxiety in autistic spectrum disorders: A behavioral and ERP study. Social Neuroscience, 10(4), 354365.

R Core Team . (2013). R: A language and environment for statistical computing.

Reed, P., Giles, A., Gavin, M., Carter, N., Osborne, L. A. (2016). Loneliness and social anxiety mediate the relationship between autism quotient and quality of life in university students. Journal of Developmental and Physical Disabilities, 28(5), 723-733. 
Renty, J. O., Roeyers, H. (2006). Quality of life in high-functioning adults with autism spectrum disorder: The predictive value of disability and support characteristics. Autism, 10(5), 511-524.

Revelle, W. (2018). psych: Procedures for personality and psychological research. https://cran.rproject.org/web/packages/psych/index.html

Rosseel, Y. (2012). lavaan: An R package for structural equation modeling. Journal of Statistical Software, 42(2), 1-36.

Rotheram-Fuller, E., Kasari, C., Chamberlain, B., Locke, J. (2010). Social involvement of children with autism spectrum disorders in elementary school classrooms. Journal of Child Psychology and Psychiatry, 51(11), 1227-1234.

Schiltz, H. K., McVey, A. J., Dolan, B. K., Willar, K. S., Pleiss, S., Karst, J. S., Carson, A. M., Caiozzo, C., Vogt, E. M., Yund, B. D. (2018). Changes in depressive symptoms among adolescents with ASD completing the PEERS $^{\circledR}$ social skills intervention. Journal of Autism and Developmental Disorders, 48(3), 834-843.

Schohl, K. A., Van Hecke, A. V., Carson, A. M., Dolan, B., Karst, J., Stevens, S. (2014). A replication and extension of the PEERS intervention: Examining effects on social skills and social anxiety in adolescents with autism spectrum disorders. Journal of Autism and Developmental Disorders, 44(3), 532-545.

Sebastian, C., Blakemore, S.-J., Charman, T. (2009). Reactions to ostracism in adolescents with autism spectrum conditions. Journal of Autism and Developmental Disorders, 39(8), 1122-1130.

Sedgewick, F., Crane, L., Hill, V., Pellicano, E. (2019). Friends and lovers: The relationships of autistic and neurotypical women. Autism in Adulthood, 1(2), 112-123.

Shattuck, P. T., Wagner, M., Narendorf, S., Sterzing, P., Hensley, M. (2011). Post-high school service use among young adults with an autism spectrum disorder. Archives of Pediatrics \& Adolescent Medicine, 165(2), 141-146.

Sosnowy, C., Silverman, C., Shattuck, P., Garfield, T. (2019). Setbacks and successes: How young adults on the autism spectrum seek friendship. Autism in Adulthood, 1(1), 44-51.

Spain, D., Sin, J., Linder, K. B., McMahon, J., Happé, F. (2018). Social anxiety in autism spectrum disorder: A systematic review. Research in Autism Spectrum Disorders, 52, 51-68.

Spence, S. H., Rapee, R. M. (2016). The etiology of social anxiety disorder: An evidence-based model. Behaviour Research and Therapy, 86, 50-67.

Sprinkle, S. D., Lurie, D., Insko, S. L., Atkinson, G., Jones, G. L., Logan, A. R., Bissada, N. N. (2002). Criterion validity, severity cut scores, and test-retest reliability of the Beck Depression Inventory-II in a university counseling center sample. Journal of Counseling Psychology, 49(3), 381-385.

Stein, M. B., Fuetsch, M., Müller, N., Höfler, M., Lieb, R., Wittchen, H.-U. (2001). Social anxiety disorder and the risk of depression: A prospective community study of adolescents and young adults. JAMA Psychiatry, 58(3), 251-256. https://doi.org/10.1001/archpsyc.58.3.251

Stice, L. V., Lavner, J. A. (2019). Social connectedness and loneliness mediate the association between autistic traits and internalizing symptoms among young adults. Journal of Autism and Developmental Disorders, 49(3), 1096-1110.

Storch, E. A., Roberti, J. W., Roth, D. A. (2004). Factor structure, concurrent validity, and internal consistency of the Beck Depression Inventory-Second Edition in a sample of college students. Depression and Anxiety, 19(3), 187-189.

Strunz, S., Schermuck, C., Ballerstein, S., Ahlers, C. J., Dziobek, I., Roepke, S. (2017). Romantic relationships and relationship satisfaction among adults with Asperger syndrome and high-functioning autism. Journal of Clinical Psychology, 73(1), 113-125.

Syu, Y.-C., Lin, L.-Y. (2018). Sensory overresponsivity, loneliness, and anxiety in Taiwanese adults with autism spectrum disorder. Occupational Therapy International, 2018, 9165978.

Toseeb, U., McChesney, G., Oldfield, J., Wolke, D. (2020). Sibling bullying in middle childhood is associated with psychosocial difficulties in early adolescence: The case of individuals with autism spectrum disorder. Journal of Autism and Developmental Disorders, 50, 14571469. https://doi.org/10.1007/s10803-019-04116-8 
Turcotte, P., Mathew, M., Shea, L. L., Brusilovskiy, E., Nonnemacher, S. L. (2016). Service needs across the lifespan for individuals with autism. Journal of Autism and Developmental Disorders, 46(7), 24802489.

Uljarević, M., Hedley, D., Rose-Foley, K., Magiati, I., Cai, R. Y., Dissanayake, C., Richdale, A., Trollor, J. (2020). Anxiety and depression from adolescence to old age in autism spectrum disorder. Journal of Autism and Developmental Disorders, 50, 3155-3165.

Weiss, R. S. (1973). Loneliness: The experience of emotional and social isolation. Massachusetts Institute of Technology Press.

White, S. W., Roberson-Nay, R. (2009). Anxiety, social deficits, and loneliness in youth with autism spectrum disorders. Journal of Autism and Developmental Disorders, 39(7), 1006-1013.

Wood, J. J., Gadow, K. D. (2010). Exploring the nature and function of anxiety in youth with autism spectrum disorders. Clinical Psychology: Science and Practice, 17(4), 281-292.

Yoo, H., Bahn, G., Cho, I., Kim, E., Kim, J., Min, J., Lee, W., Seo, J., Jun, S., Bong, G. (2014). A randomized controlled trial of the Korean version of the PEERS ${ }^{\circledR}$ parent-assisted social skills training program for teens with ASD. Autism Research, 7(1), 145-161. 